\title{
Value of distinguishing differentiated thyroid carcinoma by miRNA
}

\author{
JIANLIN XU ${ }^{1}$, DING ZHANG ${ }^{2}$, QIAN NIU $^{3}$, YONGGANG NAN ${ }^{1}$, \\ CHANGBEI SHI $^{1}$, HUA ZHAO ${ }^{4}$ and XIAOYAN LIANG ${ }^{5}$
}

\begin{abstract}
${ }^{1}$ Department of Nuclear Medicine, Shaanxi Provincial Tumor Hospital, Xi'an, Shaanxi 710061; Departments of ${ }^{2}$ Hematology and ${ }^{3}$ Pediatrics, Shaanxi Provincial People's Hospital, Xi'an, Shaanxi 710068; ${ }^{4}$ Department of Head and Neck Surgery, Shaanxi Provincial Tumor Hospital, Xi'an, Shaanxi 710061; ${ }^{5}$ Department of Radiology, Shaanxi Provincial People's Hospital, Xi'an, Shaanxi 710068, P.R. China
\end{abstract}

Received February 22, 2016; Accepted May 12, 2016

DOI: $10.3892 / 01.2016 .4557$

\begin{abstract}
Current methods for diagnosing thyroid carcinoma are time consuming or expensive. Thus, alternative approaches are required. In the present study, microRNAs (miRNAs) with higher sensitivity and specificity were screened while distinguishing between differentiated thyroid carcinoma (DTC) and subtype papillary thyroid carcinoma (PTC). A total of 120 cases suspected of having thyroid carcinoma were selected and examined using clinical color Doppler ultrasound, and computed tomography scan at the same time. The tissue specimens were obtained with fine needle aspiration, multiphase biopsy and surgical resection. The expression of miR146b, miR221 and miR222 was detected uisng the RT-quantitative polymerase chain reaction method. The receiver operating characteristic curve was used to obtain the cut-off value. Pathological examination identified 8 cases of normal thyroid tissue; 9 cases of hyperplastic nodules; 12 cases of thyroid adenoma; and 91 cases of thyroid carcinoma, of which 59 cases were DTC, 15 cases were follicular carcinoma and 17 cases were undifferentiated carcinoma. In the thyroid carcinoma, the expression levels of miR146b, miR221 and miR222 were significantly higher than those of other tissues $(\mathrm{P}<0.05)$. The expression levels of these miRNAs in the differentiated type were also significantly higher than those in the undifferentiated type $(\mathrm{P}<0.05)$. A comparison of the differentiated subunit identified no statistically significant difference $(\mathrm{P}>0.05)$. Following diagnosis of DTC, the area under curve (AUC) of miR146b, miR221 and miR222 was 0.832, 0.806 and 0.745, respectively; the cut-off values were $1.346,1.213$ and 1.425 , respectively; susceptibility was $72.8,71.5$ and $68.7 \%$, respectively; and
\end{abstract}

Correspondence to: Dr Xiaoyan Liang, Department of Radiology, Shaanxi Provincial People's Hospital, 256 West Youyi Road, Xi'an, Shaanxi 710068, P.R. China

E-mail: xiaoyan_liang1@163.com

Key words: microRNA, differentiated thyroid carcinoma, receiver operating characteristic curve, quantitative RT-PCR method specificity was $62.3,60.9$ and $59.3 \%$, respectively. The AUC of the combined miR-146b and -221 following diagnosis of PTC was 0.695 ; the cut-off values were 1.506 and 1.462 , respectively; susceptibility was $78.9 \%$; and specificity was $68.5 \%$. The AUC of the combined miR-146b and -222 was 0.677 ; the cut-off values were 1.523 and 1.443 , respectively; susceptibility was $76.3 \%$; and specificity was $66.4 \%$. The AUC of the combined miR-221 and -222 was 0.662; the cut-off values were 1.564 and 1.437 , respectively; susceptibility was $74.9 \%$; and specificity was $68.2 \%$. In conclusion, miR146b, miR221 and miR222 may be used as susceptibility and specificity indices of DTC, although they cannot be used as susceptibility or specificity indices for distinguishing PTC. Combining the two indices together can improve diagnostic accuracy to a certain extent.

\section{Introduction}

Diagnosis of thyroid neoplasm mainly depends on symptoms such as palpation, color Dopplerultrasound, endocrine hormone, fine needle aspiration (FNA) and pathology. Susceptibility of color Doppler ultrasound and computed tomography (CT) scan when diagnosing thyroid neoplasm can be $\leq 75 \%$, with a specificity of $\leq 73 \%$ (1). Multiphase FNA can improve the susceptibility of diagnosing thyroid neoplasm to $88 \%$ and specificity to $79 \%$, but it is difficult to trace (2) and requires high technology. Although pathology is the 'gold standard' for diagnostics, surgery is required to obtain specimens, delaying diagnosis and preventing early screening of tumor. Recent findings have shown that as a type of non-coding single-stranded RNA molecule coded by an endogenous gene, microRNAs (miRNAs) are closely assoicated with the occurrence and development of many diseases (3). According to analysis of the miRNA chip, the expression of miRNA146, miRNA155, miRNA187, miRNA221, miRNA222, and miRNA21 in differentiated thyroid carcinoma (DTC) were $\sim 5$ - to 10-fold higher than that of normal thyroid tissues (4). miRNA224 and miRNA339 are highly expressed in thyroid neoplasm, whereas miRNA141 was markedly decreased in Hashimoto's thyroditis (5) and miRNA154, miRNA376b and miRNA431 were markedly downregulated $\sim 5$-fold in Graves disease (6). 
Thus, miRNAs may be used as a reliable marker and applied in the diagnosis and prognostic evaluation of thyroid diseases (7). Thyroid carcinoma papillary thyroid carcinoma (PTC) has the highest incidence of thyroid carcinoma. Previous findings have identified that miR146b, miR221 and miR222 is expressed in PTC (8). In the present study, the expression conditions in various types of thyroid were examined using receiver operating characteristic (ROC) curve to compare whether single index or a combination of indices improved the susceptibility and specificity of diagnosis.

\section{Patients and methods}

Patient information. A total of 120 patients admitted to Shaanxi Provincial Tumor Hospital (Shaanxi, China) between August 2014 and August 2015 were suspected of having thyroid carcinoma, and were examined using clinical color Doppler ultrasound and CT. There were 75 males and 45 females, with an average age of $46.7 \pm 13.5$ years. Exclusion criteria for the study were hyperthyroidism, hypothyroidism, thyroiditis, Graves disease, thyroid metastatic carcinoma, women who were in treatment, gestation and lactation and no access to obtain tissue specimens. Approval for the study was obtained from the ethics committee of the Shaanxi Provincial Tumor Hospital. Written informed informed was obtained from patients and family members. To obtain tissue specimens, a FNA, multiphase biopsy or surgical resection was used. The expression of miR146b, miR221 and miR222 was detected by RT-quantitative polymerase chain reaction (RT-qPCR) method.

Detection methods. The main reagents used in the present study were: Absolute ethyl alcohol and isopropanol, chloroform (Tianjin Damao Chemical Reagent Factory, Tianjin, China), DEPC and TRIzol reagent (Invitrogen Life Technologies, Carlsbad, CA, USA), reverse transcription cDNA kit and PCR probe (Exiqon, Inc., Woburn, MA, USA).

The main instruments used included, fluorescent quantitative PCR (Rotor-Gene 6000; Qiagen, Hilden, Germany), ultraviolet spectrophotometer (Smartspec Plus 300; Bio-Rad, Berkeley, CA, USA), hypothermia high-speed centrifuge (centrifuge 2810R; Eppendorf AG, Hamburg, Germany), 96-well reverse transcription plate (Axygen Scientific, Inc., Union City, CA, USA) and $-80^{\circ} \mathrm{C}$ deep freezer (Sanyo Electric Co., Ltd., Tokyo, Japan).

According to the TRIzol reagent instructions, the one-step method was used to extract all RNAs in tissues. Agarose gel electrophoresis was used to detect its integrity and measure concentration and purity of the RNAs under an ultraviolet lamp and ultraviolet spectrophotometer. Reverse transcription was then performed. To determine the quantitative PCR reaction, samples were preserved at $-20^{\circ} \mathrm{C}$ by original $\mathrm{LNA}^{\mathrm{TM}} \mathrm{PCR}$ primer set (miR-146b, -221 and -222) and internal reference U6 reference gene primer mix. cDNA reaction product $(10 \mu \mathrm{l})$ was diluted 80 -fold, and $1.5 \mathrm{ml}$ of diluted cDNA (4 $\mu \mathrm{l})$ was mixed with SYBR-Green master mix $(5 \mu \mathrm{l})$ and primer mix $(1 \mu \mathrm{l})$ in a centrifuge tube at $11,000 \times \mathrm{g}$ for $3 \mathrm{~min}$ at room temperature and subsequently transferred to nuclease-free PCR tubes. The reaction conditions were: denaturation $95^{\circ} \mathrm{C}$ for $10 \mathrm{~min}$ (polymerase activation), annealing $95^{\circ} \mathrm{C}$ for $10 \mathrm{sec}$, and extension $60^{\circ} \mathrm{C}$ for $1 \mathrm{~min}$, with a decreasing velocity of $1.6^{\circ} \mathrm{C} / \mathrm{sec}$ and 40 cycles. Rotor-Gene 6000 Series Software 1.7 (Qiagen, Valencia, CA, USA) was used to process the data and obtain the $\mathrm{Ct}$ value. The relative quantity of sample target genes were calculated by $2^{-\Delta \Delta \mathrm{Ct}}$ with $\mathrm{U} 6$ as an internal reference and $\mathrm{H}_{2} \mathrm{O}$ as the negative control.

Statistical analysis. SPSS 19.0 statistical software (SPSS, Inc., Chicago, IL, USA) was used for data input and analysis. Measurement data were shown as the mean \pm standard deviation and comparisons between groups were analyzed by single-factor ANOVA. Independent samples t-test was used to examine comparisons between two groups. Diagnostic accuracy was analyzed by area under curve (AUC) of ROC. $\mathrm{P}<0.05$ was considered to indicate a statistically significant difference.

\section{Results}

Comparisons of miRNA expression levels between groups. There were 8 cases of normal thyroid tissue; 9 cases of hyperplastic nodules; 12 cases of thyroid adenoma; and 91 cases of thyroid carcinoma, of which 59 cases were DTC, 15 cases were follicular carcinoma and 17 cases were undifferentiated carcinoma. In thyroid carcinoma, the expression levels of miR-146b, -221 and -222 were significantly higher than those of other tissues $(\mathrm{P}<0.05)$. These indicators of differentiated type were also significantly higher than those of undifferentiated type $(\mathrm{P}<0.05)$. A comparison of the differentiated subunit, indicated no statistically significant difference (P>0.05; Fig. 1).

Susceptibility and specificity of miRNAs following diagnosis of DTC. DTC was considered as a diagnosis and others (diseases except DTC) as reference. The expression levels of miR-146b, -221 and -222 were plotted into a ROC curve. The AUC of miR-146 following diagnosis of DTC was 0.832 , when $2^{-\Delta \Delta \mathrm{Ct}}=1.346$, susceptibility was $83.2 \%$, and specificity was $79.6 \%$. The AUC of miR-221 after diagnosis of DTC was 0.806 , when $2^{-\Delta \Delta \mathrm{Ct}}=1.213$, susceptibility was $81.6 \%$, and specificity was $77.4 \%$. The AUC of miR-222 following diagnosis of DTC was 0.745 , when $2^{-\Delta \Delta \mathrm{Ct}}=1.425$, susceptibility was $78.4 \%$, and specificity was $73.9 \%$ (Fig. 2).

Susceptibility and specificity of single index and a combination of indices of miRNAs following diagnosis of PTC. PTC was considered as the diagnosis and follicular carcinoma as the reference. The expression levels of miR-146b, -221, -222 and combinations of two types were plotted into a ROC curve. The AUC of miR-146 following diagnosis of PTC was 0.644, when $2^{-\Delta \Delta \mathrm{Ct}}=1.572$, susceptibility was $72.8 \%$, and specificity was $62.3 \%$. The AUC of miR-221 following diagnosis of PTC was 0.633 , when $2^{-\Delta \Delta \mathrm{Ct}}=1.492$, susceptibility was $71.5 \%$, and specificity was $60.9 \%$; the AUC of miR-222 following diagnosis of PTC was 0.615 , when $2^{-\Delta \Delta \mathrm{Ct}}=1.448$, susceptibility was $68.7 \%$, and specificity was $59.3 \%$ (Fig. 3). The AUC of combined miR-146b and -221 following diagnosis of PTC is 0.695 , when $2^{-\Delta \Delta \mathrm{Ct}}$ was 1.506 and 1.462 , respectively, susceptibility was $78.9 \%$, and specificity was $68.5 \%$. The AUC of combined miR-146b and -222 following diagnosis of PTC is 0.677 , when $2^{-\Delta \Delta C t}$ was 1.523 and 1.443 , respectively, susceptibility was $76.3 \%$, and specificity was $66.4 \%$. The AUC 


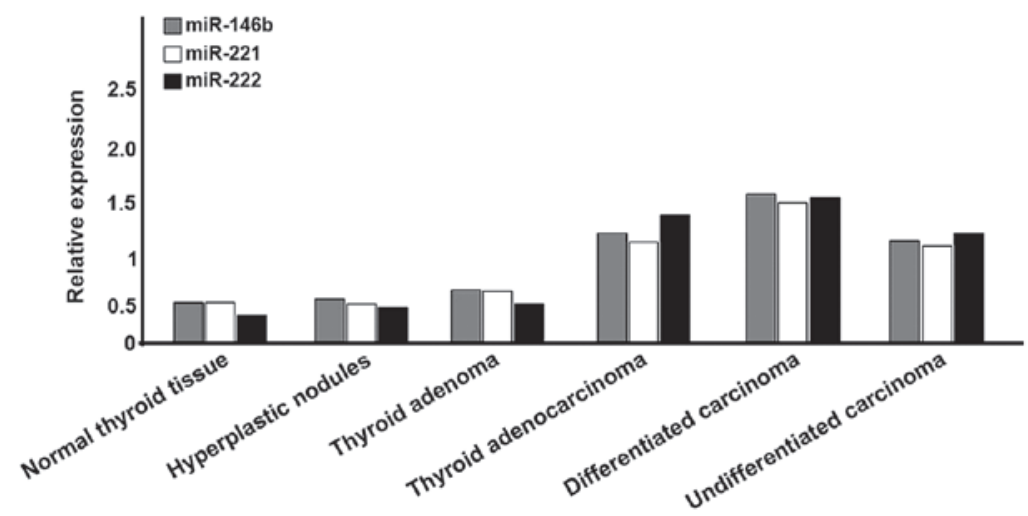

Figure 1. Relative expression of microRNAs (miRNAs), i.e., miR-146b, miR-221, miR-222 in different groups.

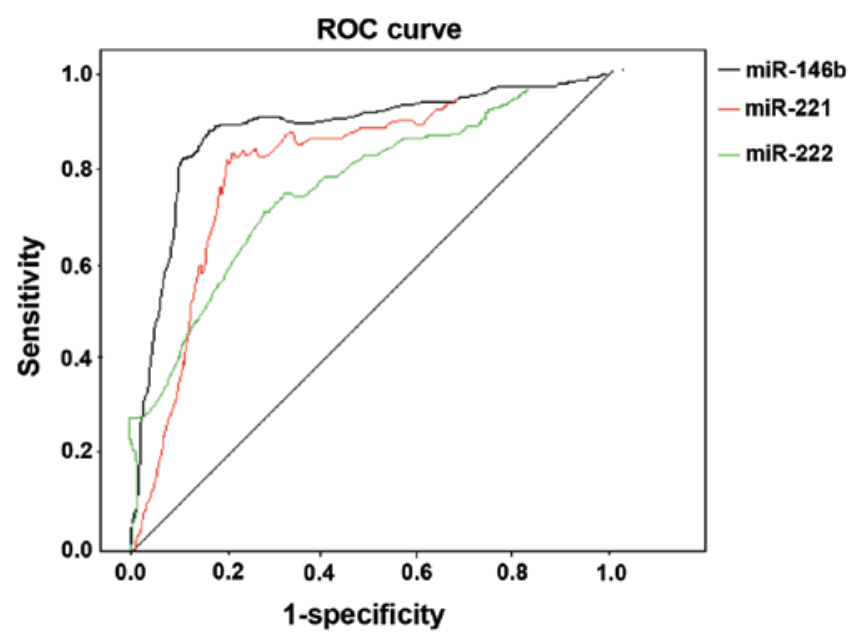

Figure 2. Receiver operating characteristic (ROC) curve between sensitivity and specificity, showing the area under the curve for miR-146b, miR-221, miR-222.

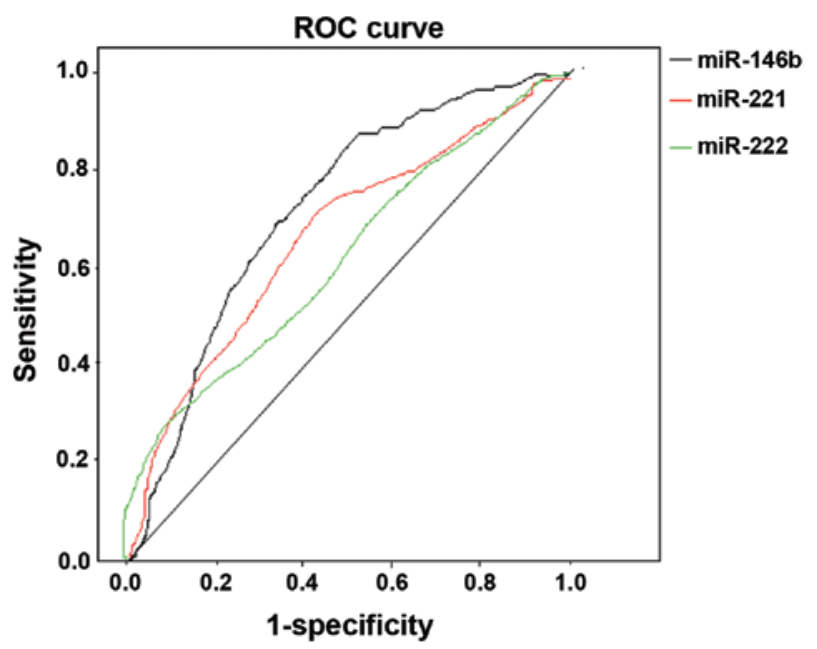

Figure 3. Receiver operating characteristic (ROC) curve between sensitivity and specificity, showing the area under the curve for miR-146b, miR-221, $\operatorname{miR}-222$.

of combined miR-221 and -222 following diagnosis of PTC is 0.662 , when $2^{-\Delta \Delta \mathrm{Ct}}$ was 1.564 and 1.437 , respectively, susceptibility was $74.9 \%$, and specificity was $68.2 \%$ (Fig. 4).

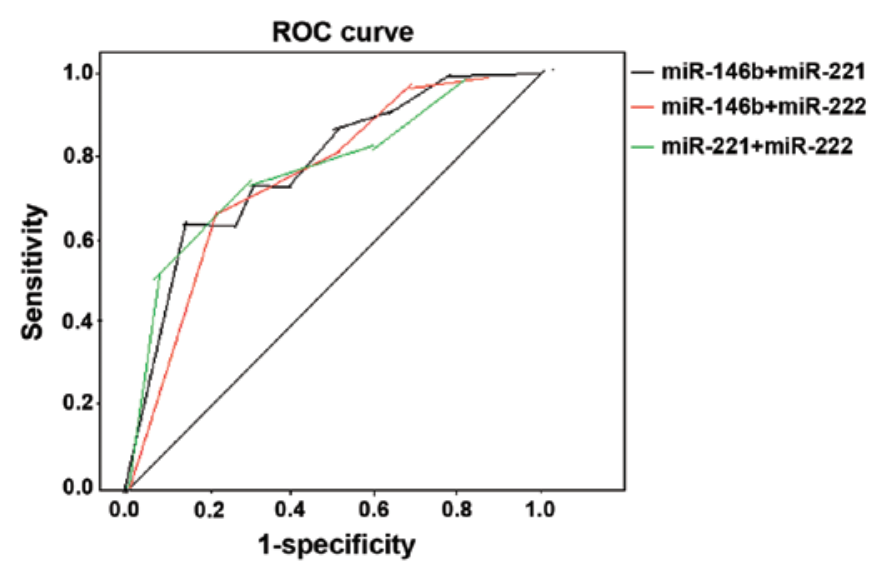

Figure 4. Receiver operating characteristic (ROC) curve between sensitivity and specificity, showing the area under the curve for combinations of miR-146b, miR-221, miR-222.

\section{Discussion}

Mature miRNA engages in the formation of RNA-induced silencing complex, known as the miRNA ribonucleoprotein complex (7). In the majority of cases, single-stranded miRNA of compounds and 3'UTR of its homologous mRNA have incomplete complementary pairing, which interrupts translation of this gene and regulates the protein expression. In addition, miRNA and its mRNA have complete complementary pairing, which leads to a specific fracture of target mRNA in the complementary region and gene silencing (9). This feature of miRNA can be used as a sensitive indication during the diagnosis of diseases in the early stage (10).

Chen et al (11) identified that compared with follicular adenoma, PTC markedly upregulates miR-146b, -221 and -222, while no difference was identified for the expression of $-146 a$, -155 and -187 . Compared with non-PTC, including poorly differentiated adenocarcinoma, hyperplastic nodules and normal thyroid tissue, miR-146b was highly expressed in PTC while there was no distinctive difference in the subtypes of DTC. Nikiforova et al (12) also compared BRAF, RAS and RET, three types of different mutations of PTC, and identified that miR-187 and -146b expression was downregulated in the RAS and BRAF mutational group, while this expression was upregulated in the RET mutational group, with the expression 
levels of the BRAF and RAS mutational groups being the highest (12). Through flat plate clone test, it was found that compared with the control group, clone numbers of PTC cells of transfected miR-221 were more than double (13). Conversely, by calculating cells at 72 and $96 \mathrm{~h}$ after knocking out miR-221, it was found that cell counts decreased $\sim 0.3$-fold compared with the control group. Thus, the expression of miR-221 may be significantly associated with the cell proliferation of PTC. Follicular adenocarcinoma (FTC) is only $10 \%$ of DTC. Colamaio et al (14) found that compared with normal thyroid tissues, the expression of miR-191 significantly decreased in FTC, and that upregulated miR-191 in the WRO cell line of FTC, not only changes cell morphology, but also inhibits cyclin CDK6, and blocks cells in the G1 period, which inhibits cell proliferation and migration.

Based on the above results, the present study identified that miR-146b,-221 and -222 are highly expressed in DTC, although there was no difference in the subtypes of DTC. According to the ROC curve, single miR-146b, -221 or -222 can be used as higher indices of susceptibility and specificity while diagnosing DTC, but single index does not perform well while distinguishing PTC, and combinations of the two types improved diagnostic accuracy, and there was no distinctive difference. However, compared with the single index while diagnosing DTC, susceptibility and specificity were not optimal; thus, there was no distinctive difference between miR-146b, -221 or -222 in the different subtypes of DTC. The significance of the different expressions of miRNA in various diseases remains to be determined. In addition, how it regulates and controls cell proliferation, migration and differentiation in various types of tumor and specific cell signaling pathways has yet to be determined. Thus, whether different subtypes of DTC have miRNAs of obvious differential expression remains to be elucidated.

\section{References}

1. Wu Q, Li Y and Wang Y: Diagnostic value of 'absent' pattern in contrast-enhanced ultrasound for the differentiation of thyroid nodules. Clin Hemorheol Microcirc: Dec 15, 2015 (Epub ahead of print).
2. Fang Q, Cai $\mathrm{C}$ and Chen $\mathrm{H}$ : Application value of fine needle aspiration and cell block in preoperative diagnosis of thyroid cancer and discrimination of follicular tumor. Zhonghua Er Bi Yan Hou Tou Jing Wai Ke Za Zhi 50: 668-672, 2015 (In Chinese).

3. Kouniavsky G and Zeiger MA: The quest for diagnostic molecular markers for thyroid nodules with indeterminate or suspicious cytology. J Surg Oncol 105: 438-443, 2012.

4. He H, Jazdzewski K, Li W, Liyanarachchi S, Nagy R, Volinia S, Calin GA, Liu CG, Franssila K, Suster S, et al: The role of microRNA genes in papillary thyroid carcinoma. Proc Natl Acad Sci USA 102: 19075-19080, 2005.

5. Dorris ER, Smyth P, O'Leary JJ and Sheils O: MIR141 expression differentiates Hashimoto thyroiditis from PTC and benign thyroctytes in Irish archival thyroid tissues. Front Endocrinol (Lausanne) 3: 102, 2012.

6. Liu R, Ma X, Xu L, Wang D, Jiang X, Zhu W, Cui B, Ning G, Lin D and Wang S: Differential microRNA expression in peripheral blood mononuclear cells from Graves' disease patients. J Clin Endocrinol Metab 97: E968-E972, 2012.

7. Graham ME, Hart RD, Douglas S, Makki FM, Pinto D, Butler AL, Bullock M, Rigby MH, Trites JR, Taylor SM, et al: Serum microRNA profiling to distinguish papillary thyroid cancer from benign thyroid masses. J Otolaryngol Head Neck Surg 44: 33, 2015.

8. Chou CK, Chen RF, Chou FF, Chang HW, Chen YJ, Lee YF, Yang KD, Cheng JT, Huang CC and Liu RT: miR-146b is highly expressed in adult papillary thyroid carcinomas with high risk features including extrathyroidal invasion and the BRAF(V600E) mutation. Thyroid 20: 489-494, 2010.

9. Connerty P, Ahadi A and Hutvagner G: RNA Binding Proteins in the miRNA Pathway. Int J Mol Sci 17: 17, 2015.

10. Lee JC, Zhao JT, Gundara J, Serpell J, Bach LA and Sidhu S: Papillary thyroid cancer-derived exosomes contain miRNA-146b and miRNA-222. J Surg Res 196: 39-48, 2015.

11. Chen YT, Kitabayashi N, Zhou XK, Fahey TJ III and Scognamiglio T: MicroRNA analysis as a potential diagnostic tool for papillary thyroid carcinoma. Mod Pathol 21: 1139-1146, 2008.

12. Nikiforova MN, Tseng GC, Steward D, Diorio D and Nikiforov YE: MicroRNA expression profiling of thyroid tumors: Biological significance and diagnostic utility. J Clin Endocrinol Metab 93: 1600-1608, 2008.

13. Pallante P, Visone R, Ferracin M, Ferraro A, Berlingieri MT, Troncone G, Chiappetta G, Liu CG, Santoro M, Negrini M, et al: MicroRNA deregulation in human thyroid papillary carcinomas. Endocr Relat Cancer 13: 497-508, 2006.

14. Colamaio M, Borbone E, Russo L, Bianco M, Federico A, Califano D, Chiappetta G, Pallante P, Troncone G, Battista S, et al: miR-191 down-regulation plays a role in thyroid follicular tumors through CDK6 targeting. J Clin Endocrinol Metab 96: E1915-E1924, 2011. 